\title{
Correction to: Interlaboratory Reproducibility of Contour Method Data Analysis and Residual Stress Calculation
}

\author{
C.R. D'Elia ${ }^{1}$ - S.S. Carlson ${ }^{2}$ - M.L. Stanfield ${ }^{2}$ - M.B. Prime ${ }^{3}$ • J. Araújo de Oliveira ${ }^{4}$. \\ T.J. Spradlin ${ }^{5} \cdot$ J.B. Lévesque ${ }^{6} \cdot$ M.R. Hill $^{1}$
}

Published online: 17 June 2020

(C) Society for Experimental Mechanics 2020

\section{Correction to: Experimental Mechanics https://doi.org/10.1007/s11340-020-00599-0}

The original version of this article has been corrected to include authors middle initials in the author list. The details given in this correction are correct.

The online version of the original article can be found at https://doi.org/ 10.1007/s11340-020-00599-0

M. R. Hill

mrhill@ucdavis.edu

1 Department of Mechanical and Aerospace Engineering, University of California, One Shields Avenue, Davis, CA 95616, USA

2 Mechanical Engineering, Aerospace Structures Section, Southwest Research Institute (SwRI), 6220 Culebra Rd, San Antonio, TX 78238, USA

3 Los Alamos National Laboratory, Los Alamos, NM 87545, USA

4 StressMap, Engineering \& Innovation, Open University, N2024, Venables Bldg. Walton Hall, Milton Keynes MK7 6AA, UK

5 United States Air Force, Air Force Research Laboratory, 2790 D. St. Wright-Patterson, AFB, Fairborn, OH 45433, USA

6 Hydro-Québec Research Institute, 1800, Lionel-Boulet, Varennes, Québec J3X 1S1, Canada 